


\title{
Tuberkulose im Rettungsdienst Infektionsrisiko durch Flüchtlinge?
}

\author{
Tuberkulose ist hierzulande selten. Mit der zuletzt starken Zuwanderung \\ nach Deutschland wächst aber die Furcht vor einer Wiederverbreitung \\ dieser Erkrankung. Gerade die Mitarbeiter, die bei der Versorgung der \\ Flüchtlinge helfen, könnten einer erhöhten Infektionsgefahr ausgesetzt \\ sein. Das stimmt - das Risiko lässt sich aber gut kontrollieren.
}

Thomas Jakob • Jens Tiesmeier

Flüchtlinge haben häufiger Tuberkulose Die Zahl der Migranten und Asylsuchenden aus Krisengebieten hierzulande steigt stetig. Da viele Flüchtlinge aus Ländern mit unzureichender medizinischer Versorgung und mangelhaften hygienischen Verhältnissen stammen, besteht die Möglichkeit, dass Rettungsdienste häufiger als bisher mit (Verdachts-)Fällen einer Tuberkulose (Tbc) konfrontiert werden - z. B. bei Einsätzen in Gemeinschaftsunterkünften. Daten zum Tuberkulose-Screening bei Asylbewerbern einer Aufnahmeeinrichtung aus Trier stützen diese Annahme ( $\bullet$ Abb. 1):

- Für die Jahre 2001-2014 gab das zuständige Gesundheitsamt dort die Tuberkulose-Aufnahmeprävalenz mit 305/100000 Untersuchten an - bei einer bundesweiten Prävalenz in Deutschland von 7,5/100000 Einwohnern [20].

- Zudem lag die Aufnahmeprävalenz unter den Asylbegehrenden deutlich höher als die Tuberkuloseprävalenzen der jeweiligen Heimatländer.

Angst vor Ansteckung bei Helfern Hieraus kann bei Mitarbeitern im Rettungsdienst eine subjektiv empfundene Unsicherheit entstehen - bezüglich des infektiologisch korrekten Umgangs mit solchen Patienten und den hieraus resultierenden notwendigen hygienischen Maßnahmen.

- Im eigenen Rettungsdienstbereich wurde das Risiko für eine Ansteckung vielfach als angstbehaftet bewertet.

Zur Versachlichung des Themas sollen die nachfolgenden Informationen einen kurzen Überblick über rettungsdienstlich relevante Aspekte der Tuberkulose geben. Maßnahmen der Standardhygiene sowie Aspekte einer speziellen (laborchemischen und bakteriellen) Diagnostik, die differenzierte Therapie und Hygienemaßnahmen in der Klinik sind nicht Gegenstand dieses Artikels.

\section{Informationen zur Tuberkulose-Erkrankung}

Erreger Der häufigste Erreger der Lungentuberkulose ist das Mycobacterium tuberculosis, typus hominis. Mykobakterien sind aerobe, stäbchenförmige Bakterien, die aufgrund ihrer Färbeeigenschaften zur Mikroskopie als „säurefest“ bezeichnet werden. Infektionen mit M. bovis (Rindertuberkulose) sind mittlerweile selten, weitere Erreger des M.-tuberculosis-Komplexes Einzelfälle [1, 2].

Epidemiologie Mit geschätzt 9 Mio. erkrankten Menschen(WHO 2013) ist die Tuberkulose eine der häufigsten Infektionskrankheiten weltweit [20].

Abb. 1 Anzahl der Tuberkulosefälle in Rheinland-Pfalz in den Jahren 2009-2014. Dargestellt sind die Gesamtzahlen und der Anteil, der davon auf Flüchtlinge zurückgeht (Daten des Gesundheitsamts der Kreisverwaltung Trier-Saarburg, aus [20]).

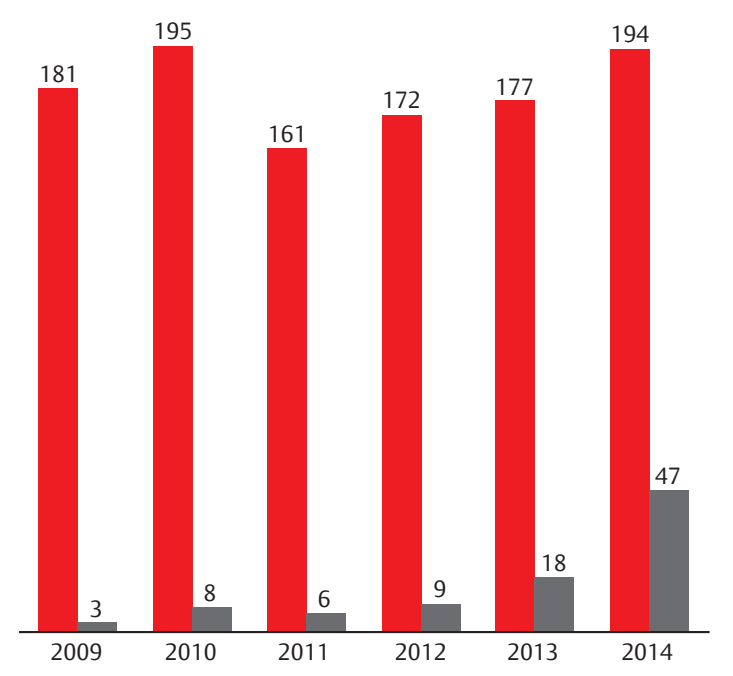




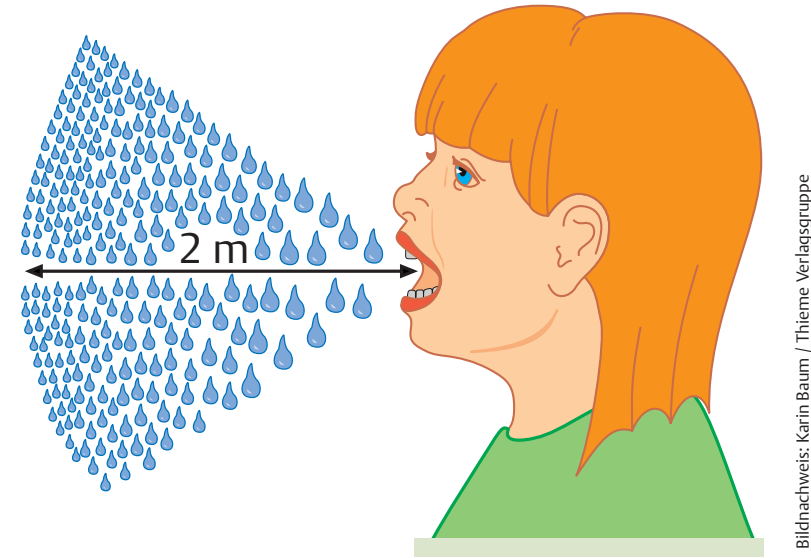

Abb. 2 Atemaerosole verbreiten sich über eine Distanz von mehreren Metern. Die Freisetzung erfolgt einfach - über Niesen, Husten oder durch feinste Tröpfchen beim Sprechen.

- Deutschland gehört zu den Niedriginzidenzländern: Die Zahl der Neuerkrankungen lag im Jahr 2013 bei 4318 (Inzidenz: 5,4/100000 Einwohnern), wobei der Anteil multiresistenter Erreger 2,1\% betrug [20].

- $62 \%$ der Patienten waren männlich, 38\% weiblich.

- Unterschieden nach der Staatsangehörigkeit waren $52 \%$ deutsche und $48 \%$ ausländische Staatsangehörige. $43 \%$ der Patienten wurden in Deutschland und 56\% im Ausland geboren.

- Zwei Erkrankungshäufungen sind zu verzeichnen: Eine in der Altersgruppe 20-29 Jahre und eine weitere bei Senioren ab dem 69. Lebensjahr.

Während die Inzidenz in Westeuropa im Durchschnitt unter15/100 000 Einwohnern liegt, ist sie in den Balkanstaaten mit 20/100000 und in Osteuropa mit 100/100 000 Einwohnern im Vergleich erhöht [1, 2, 20].

Verbreitung über Aerosole Eine Infektion geht in der Regel von Patienten mit offener Lungentuberkulose aus - bei denen also ein Herd Anschluss an die Luftwege hat [3].

- Die Übertragung erfolgt fast immer von Mensch zu Mensch und aerogen, d.h. durch feinste erregerhaltige Tröpfchenkerne $(<5 \mu \mathrm{m})$.

Erkrankte Personen setzen diese insbesondere beim Husten und Niesen frei (aber auch beim Lachen, lauten Singen und Sprechen, - Abb. 2).

Neuere Untersuchungen ergaben, dass ein „face-to-face“-Kontakt von $>20$ min nötig ist, um sich anzustecken.
Andere Übertragungswege eher unbedeutend Wenn von einer niedrigen Erregerkonzentration auszugehen ist und es nicht zur Aerosolbildung kommt, ist die Übertragungswahrscheinlichkeit gering.

- Selbst eingetrocknetes Sputum auf Oberflächen wird - trotz der langen Überlebensfähigkeit der Erreger darin - nicht als relevante Infektionsquelle erachtet [2, 4-6 ].

Auch bei extrapulmonaler Tbc kann es zur Ausscheidung von Erregern kommen. Dann jedoch über Urin, Eiter, Perforation von Lymphknoten und Herden bzw. Wundsekret.

Längere Inkubationszeit Die Zeit zwischen Infektion und einer messbaren Immunantwort beträgt im Durchschnitt 6-8 Wochen. Nur ein geringer Teil der Infizierten erkrankt tatsächlich an einer behandlungsbedürftigen Tbc (5-10\% der immunkompetenten Jugendlichen und Erwachsenen, davon die Hälfte innerhalb der ersten 2-3 Jahre) [2].

Risiko einer Ansteckung Die Ansteckungsfähigkeit an offener Lungen-Tbc Erkrankter ist am höchsten,

- wenn im Sputum mikroskopisch säurefeste Stäbchen nachweisbar sind.

- Sie ist wesentlich geringer, wenn nur ein kultureller oder molekularbiologischer Nachweis gelingt [2].

Zur Ansteckungsfähigkeit gehört dann auch eine Symptomatik, die es ermöglicht, die Erreger dermaßen in die Raumluft zu befördern, dass eine Aufnahme durch andere Personen über die Einatemluft möglich ist, d.h. Husten, Niesen etc. [3].

- Kinder unter 10 Jahren gelten in aller Regel als nicht infektiös (häufig mikroskopisch negativ, schwacher Hustenstoß).

- Patienten, die klinisch auf eine Therapie ansprechen (Rückgang der Hustensymptomatik), sind in der Regel nach 3 Wochen nicht mehr ansteckend $[1,7,8]$.

Bei Kavernenbildung und Resistenz kann eine deutlich längere Ansteckungsfähigkeit bestehen und selbst bei adäquater Behandlung in bis zu 10\% der Patienten ein mikroskopisch positiver Sputumbefund erhoben werden [1].

Typisches Symptom: Husten Die Manifestation äußert sich bei ca. $80 \%$ als Lungen-Tbc, prinzipiell kann aber jedes Organ befallen werden. Häufig bestehen keine charakteristischen Beschwerden.

- Leitsymptom der Lungen-Tbc ist Husten mit oder ohne Auswurf (selten blutig, dann ist eine sofortige Abklärung nötig).

- Gelegentlich kommt es zu Brustschmerz und Atemnot. 
Jeder länger als 3 Wochen bestehende Husten sollte abgeklärt werden.

Ergänzende Symptomatik Allgemeinsymptome sind Einschränkungen des Befindens, Appetitmangel, Gewichtsabnahme, leichtes Fieber, vermehrtes Schwitzen (besonders nachts), Müdigkeit, Schwäche und Grippesymptome.

Kinder sind in über $50 \%$ der Fälle asymptomatisch.

Extrapulmonale Tbc Insbesondere bei Einschränkung der Immunabwehr kann es zu einer lymphogenen oder hämatogenen Ausbreitung mit Befall weiterer Organe kommen (primäre Generalisation).

- Dabei sind besonders Säuglinge und Kleinkinder durch eine primäre hämatogene Aussaat gefährdet, da ihre Immunabwehr noch nicht voll ausgebildet ist.

- Hauptkomplikationen sind die Miliartuberkulose und die tuberkulöse Meningitis.

Durch spätere Reaktivierung und hämatogene Aussaat können nach Jahren noch Knochen-, Gelenks- und Urogenitaltuberkulosen auftreten (postprimäre Tbc). Die Symptomatik ist dann organspezifisch [2].

\section{Infektionsgefahr im Rettungsdienst}

Risikofaktoren Belastbare Daten, ob auch Beschäftigte im Rettungsdienst in Deutschland erkrankt sind, liegen nicht vor [9]. Die Ansteckungsfähigkeit ist jedoch eher gering und nicht so leicht möglich wie bei anderen aerogen übertragbaren Krankheiten (z.B. Masern, Varizellen). Sie hängt von folgenden Faktoren ab:

- Häufigkeit, Dauer und Enge des Kontakts (>8h kumulativ im geschlossenen Raum oder intensiver Kontakt, besonders bei noch nicht diagnostizierten Kranken und fehlenden Schutzmaßnahmen).

- Menge und Virulenz der inhalierten Erreger

- Empfänglichkeit der disponierten Person (Immunschwäche) $[2,10,11]$.

Es ist davon auszugehen, dass die Mehrzahl der Mitarbeiter eine Immunität im Sinn einer „stillen Feiung“ erworben hat. Das bedeutet, dass Kontakte mit Tuberkulose ohne Erkrankung zu einer Immunität führen.
Hier besteht ein erhöhtes Risiko Diagnostische und therapeutische Tätigkeiten mit engem Kontakt zu ansteckungsfähigen Personen (insbesondere vor Diagnosestellung) erhöhen die Gefahr einer Erregerübertragung. Dies sind v.a.

- Intubation,

- Absaugen,

- Reanimation und

- Verzicht auf gezielte Infektionsschutzmaßnahmen $[1,13]$.

Patienten mit geschlossener Beatmung stellen jedoch kein erhöhtes Risiko dar [12].

So können Sie sich schützen Die wirksamste Prophylaxe vor Ansteckung besteht darin, sich vor der Inhalation infektiöser Aerosole zu schützen und die unten beschriebenen Maßnahmen einzuhalten. Die früher übliche BCG-Impfung (BCG: Bacillus CalmetteGuérin) ist inzwischen eingestellt, weil Lücken in der Wirksamkeit beschrieben sind.

Infektionskategorie bestimmt Hygienemaßnahmen Hygieneund Vorsichtsmaßnahmen ergeben sich aus der Einteilung der Patienten in Kategorien gemäß AWMF [1, 4] (s. auch retten! 4/15).

- Kategorie A: Kein Anhalt für Vorliegen einer Infektionskrankheit.

- Kategorie B: Infektion besteht und ist diagnostiziert, kann aber nicht durch beim Transport übliche Kontakte übertragen werden (Virushepatitis, HIV, Tuberkulose exklusive offene LungenTbc).

$\triangleright$ Ausnahme: offene und blutende Wunden (hier Kategorie C oder D).

- Kategorie C-I: Gesicherte Diagnose oder begründeter Verdacht auf kontagiöse Infektionskrankheit (offene Lungen-Tbc, Meningokokken-Meningitis),

$\triangleright$ auch Patienten mit multiresistenten Keimen (Methicillinresistenter Staphylococcus aureus [MRSA], Vancomycinresistente Enterokokken [VRE], multiresistente gramnegative Stäbchen [4-MRGN]) und

$\triangleright$ Patienten, die akut erbrechen und / oder dünnflüssige Stühle ausscheiden.

- Kategorie C-II: Bloßer Verdacht auf Infektionskrankheit mit besonders gefährlichen Erregern (Hämorrhagisches Fieber, SARS).

- Kategorie D: Patienten, die in hohem Maße infektionsgefährdet sind (Polytrauma, ausgedehnte Verbrennungen, Immunsuppression).

Infektionskategorie der Tbc Nach AWMF fallen Tbc-Patienten in die Kategorie B oder (bei offener Lungen-Tbc) in die Kategorie C-I. Die Technische Regel für Biologische Arbeitsstoffe (TRBA 250) stuft Patienten mit offener Lungen-Tbc in die Risikogruppe 3 ein 
Tab. 1 Übersicht über Eigenschaften verschiedener Tuberkulose-Formen und Schutzmaßnahmen gegen eine Ansteckung

\begin{tabular}{|c|c|c|}
\hline & Offene Lungen-Tbc & Tbc, übrige Formen \\
\hline Definition & $\begin{array}{l}\text { Gewebseinschmelzung des Lungenparenchyms } \\
\text { mit Öffnung nach außen }\end{array}$ & Befall/Gewebseinschmelzung verschiedener Organe \\
\hline Erreger & \multicolumn{2}{|c|}{ Mycobacterium tuberculosis } \\
\hline infektiöse Substanzen & $\begin{array}{l}\text { Atemwegssekrete, kontaminierte Staubpartikel } \\
\text { (wenn sie in aerosolierte Form gebracht werden) }\end{array}$ & $\begin{array}{l}\text { je nach Lokalisation: Eiter, Urin, Stuhl, Liquor, Blut, } \\
\text { genitaler Ausfluss }\end{array}$ \\
\hline Übertragungsweg & aerogen & iatrogen, direkte und indirekte Kontakte \\
\hline persönliche Schutzausrüstung (PSA)* & $\begin{array}{l}\text { Schutzhandschuhe } \\
\text { Schutzkittel } \\
\text { - FFP2-Maske (DIN EN 149) }\end{array}$ & $\begin{array}{l}\text { Schutzhandschuhe } \\
\text { Schutzkittel }\end{array}$ \\
\hline Desinfektion & $\begin{array}{l}\text { Tuberkulozid } \\
\text { Koutinedesinfektion der patientennahen (und) } \\
\text { Kontaktflächen } \\
\text { - Schlussdesinfektion: gesamter Innenraumbereich ** }\end{array}$ & $\begin{array}{l}\text { Tuberkulozid } \\
\text { Routinedesinfektion der patientennahen (und) } \\
\text { Kontaktflächen }\end{array}$ \\
\hline kontaminierte Abfälle & \multicolumn{2}{|c|}{$\begin{array}{l}\text { - infektiös (AS } 180 \text { 103), Entsorgung über Krankenhaus } \\
\text { - Atemschutz gilt - sofern nicht direkt kontaminiert - nicht als infektiöser Abfall (Entsorgung nach AS } 180 \text { 104) }\end{array}$} \\
\hline kontaminierte Wäsche & \multicolumn{2}{|c|}{ Entsorgung als infektiöse Wäsche } \\
\hline \multicolumn{3}{|c|}{$\begin{array}{l}\text { *PSA: konkret zu tragende PSA ist auf die jeweiligen Sachverhalte abzustimmen (z. B.: patientennahe Tätigkeiten, Übertragungswege, Immunstatus der Beschäftigten). } \\
\text { Eine FFP-Maske kann sich für den Betreuenden erübrigen, wenn der Patient in der Lage ist, eine entsprechende Maske zu tragen. Die Empfehlungen verschiedener Fachgesellschaf } \\
\text { ten variieren aufgrund mangelnder wissenschaftlicher Evidenz zur Wirksamkeit verschiedener Arten von Atemschutz in der Infektionsprävention erheblich [1]. } \\
\text { Der FFP2-Standard ist eine Mindest-Empfehlung. Natürlich kann auch eine FFP3-Maske genutzt werden. } \\
{ }^{* *} \text { Grundsätzlich reichen Maßnahmen der Standardhygiene. Geeignetes, d. h. Desinfektionsmittel mit nachgewiesener Wirkung auch gegen Mykobakterien verwenden } \\
\text { (s. VAH-Liste) [19]. Bei großflächiger Kontamination durch stark hustenden Patienten ohne Mund-Nasen-Schutz ist im Anschluss an den Transport eine Wischdesinfektion aller } \\
\text { möglichen Kontaktflächen vorzunehmen. }\end{array}$} \\
\hline
\end{tabular}

(Risiko bei Tätigkeiten wie Absaugen, Intubation, Hustenprovokation) - mit Maßnahmen der Schutzstufe 3 (z.B. FFP2-Maske) [14].

Konkret: Schutz bei (offener) Tuberkulose Fällt Ihr Patient in die Kategorie B, genügt es, die Standardhygiene einzuhalten. Für Patienten der Kategorie C-I und bekanntem Infektionsstatus empfiehlt sich dagegen folgendes Vorgehen (s. auch $\bullet$ Tab. 1):

- Alle Besatzungsmitglieder und Zieleinrichtung informieren.

- Fahrerabteil getrennt halten, Zwischenfenster schließen, Umluft ausschalten.

- Anlegen eines Mund-Nasen-Schutzes beim Patienten [15], sofern er dies toleriert.

- Schutzkleidung tragen (Haube, Handschuhe, Schutzkittel, ggf. FFP2-Maske [5]).

- Flächen, die mit Sekreten kontaminiert werden, sofort desinfizieren.

- Einmalmaterialien verwerfen - auch bei bloßem Verdacht der Kontamination.

- Reinigung und Desinfektion des Fahrzeugs vor Ablegen der Schutzkleidung.

- Nach Transport: Wischdesinfektion aller Flächen (ggf. Rücksprache mit Gesundheitsamt).
Beim Transport steht der Atemschutz im Vordergrund [3]. Lüften kann die Zahl infektiöser, schwebender Partikel erheblich senken: Wenn möglich, Lüftung/Klimaanlage beim Transport ausschalten und Fenster öffnen (cave: Auskühlung der Patienten).

Gegenstände aus Patientenraum entfernen oder nicht? Oft heißt es, man solle zum Schutz den Patientenbereich soweit wie möglich ausräumen. Das ist unnötig. Geschlossene Behältnisse stellen eine ausreichende Barriere dar. Koffer, Rucksäcke etc., die ohne Halterung im Fahrerraum liegen, sind vielmehr ein Unfallrisiko.

Das richtige Desinfektionsmittel verwenden Beim Desinfizieren ist darauf zu achten,

- dass das Desinfektionsmittel hinsichtlich der Konzentration und Chemie auf Tuberkulozidie vom Verband für angewandte Hygiene (VAH) zertifiziert ist.

Eine Raumluftdesinfektion, bei der Desinfektionsmittel versprüht oder Formalin verdampft wird, ist üblicherweise nicht erforderlich [16]. Sie kommt allenfalls auf Anordnung der Gesundheitsämter nach $§ 18$ IfSG bei multiresistenten Lungentuberkulosen vor.

Kategorie C-I, Infektionsstatus erst am Abholort bekannt Erfährt man erst am Einatzort, dass der Patient an offener Tbc erkrankt ist, sollte man folgendes tun: 
- Bei einem Notfalltransport prüfen Sie zunächst die Dringlichkeit. Ist diese gegeben, führen Sie den Transport durch. Berücksichtigen Sie dabei die oben angegebenen Schutzmaßnahmen.

Das Patientenleben hat unter Wahrung von Selbstschutz des Personals Vorrang!

- Handelt es sich um einen elektiven Krankentransport, planen Sie die Maßnahme, und führen Sie sie unter Berücksichtigung der genannten Schutzmaßnahmen durch.

Wenn Sie Schutzkleidung tragen: Achten Sie besonders auf das kontaminationsfreie Ablegen (s. auch [22])!

Was tun bei Verdacht auf Kontamination des Personals? Selten erfährt man nachträglich vom vorher unbekannten Tbc-Status der transportierten Person. Eine Infektion kann eigentlich nur geschehen, wenn ungeschützt erregerhaltige Aerosole eigeatmet werden.

- Informieren Sie im Zweifel den unmittelbaren Dienstvorgesetzten und erstellen Sie ein Gedächtnisprotokoll.

- Der ÄLRD kann zur Konsultation kontaktiert werden.

- Bei Fragen zum weiteren Vorgehen kann dann das Gesundheitsamt beraten und ggf. Anordnungen treffen.

Grundsätzlich besteht aufgrund der langen Inkubationszeit und der währenddessen fehlenden Ansteckungsfähigkeit kein „notfallmäßiger“ Handlungsbedarf. Personal im Rettungsdienst muss also bei Verdacht auf Kontakt mit dem Erreger nicht sofort und automatisch außer Dienst gestellt werden.

Meldepflicht Der feststellende Arzt ist nach Infektionsschutzgesetz (IfSG $\S 6$ Abs. 1) verpflichtet, Erkrankung sowie Tod an einer behandlungsbedürftigen Tuberkulose zu melden [18]. Der Rettungsdienst ist nach $\S 8(2)$ IfSG von der Meldepflicht befreit, wenn der Patient einer ärztlich geleiteten Einrichtung übergeben wird.

\section{Fazit}

In Deutschland ist das Risiko einer Tuberkuloseinfektion gering. Die Gefahr einer Infektion steigt mit der Kontaktdauer und -häufigkeit mit ansteckungsfähigen Patienten an. Anstelle von unbegründeter Furcht oder Vorbehalten im Umgang mit Patienten mit Migrationshintergrund ist ein wachsamer Umgang mit Verdachtsfällen ratsam. Hierbei steht der Schutz vor der Inhalation infektiöser Aerosole, wie sie durch Husten oder bei Maßnahmen des Airwaymanagements entstehen können, im Vordergrund - besonders durch Nutzen der persönlichen Schutzausrüstung und Anlegen eines Mund-Nasen-Schutzes beim Patienten.
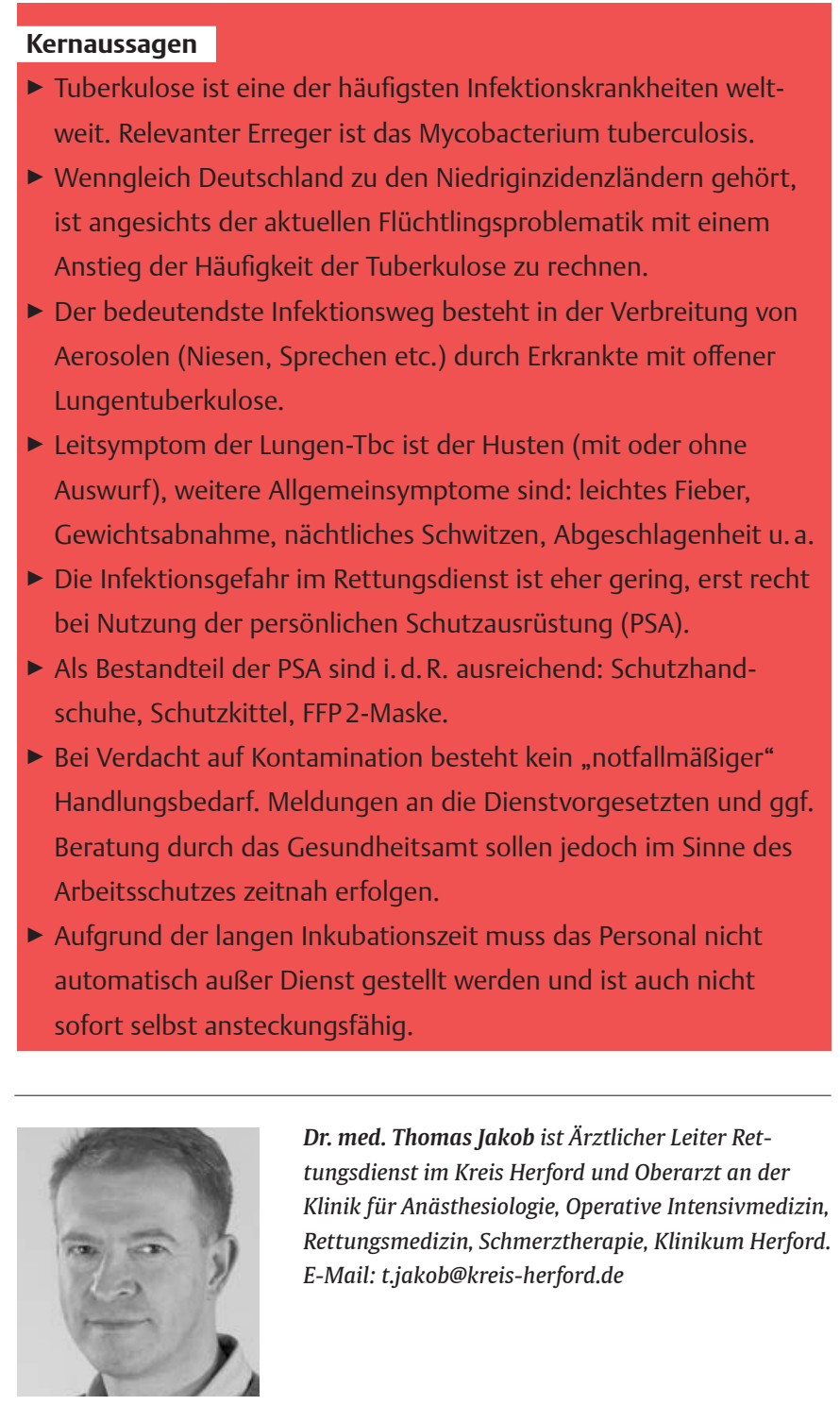

Dr. med. Thomas Jakob ist Ärztlicher Leiter Rettungsdienst im Kreis Herford und Oberarzt an der Klinik für Anästhesiologie, Operative Intensivmedizin, Rettungsmedizin, Schmerztherapie, Klinikum Herford. E-Mail: t.jakob@kreis-herford.de

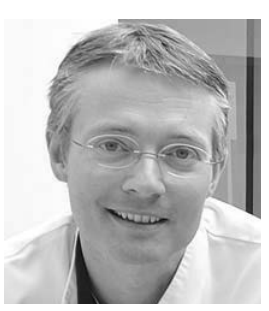

Dr. med. Jens Tiesmeier ist Ärztlicher Leiter am Notarztstandort Krankenhaus Bad Oeynhausen. Er arbeitet als Oberarzt am Institut für Anästhesiologie und Intensivmedizin der MKK-Mühlenkreiskliniken in Bad Oeynhausen.

E-Mail:Jens.Tiesmeier@muehlenkreiskliniken.de

\section{Infos im Internet}

Das Literaturverzeichnis zu diesem Beitrag finden Sie im Internet: Rufen Sie unter www.thieme-connect.de/products die Seite von retten! auf und klicken Sie beim jeweiligen Artikel auf „Zusatzmaterial“. 


\section{Tuberkulose im Rettungsdienst Infektionsrisiko durch Flüchtlinge?}

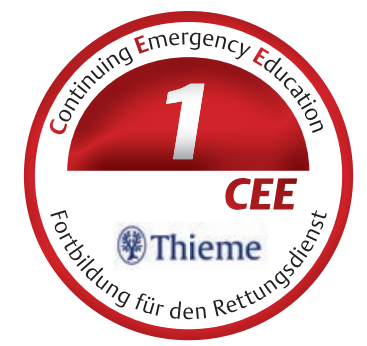

1 Welche Aussage zur Häufigkeit der Tuberkulose (Tbc) stimmt?

A In Deutschland ist die Zahl der Neuerkrankungen besonders hoch.

B Tuberkulose ist weltweit eine eher unbedeutende Infektionserkrankung.

C Erwachsene zwischen 20 und 29 Jahren sind häufiger betroffen als 30- bis 39-jährige.

D Fast alle (80-90\%) Erkrankten in Deutschland sind Ausländer.

E In Deutschland erkranken Frauen häufiger an Tbc als Männer.

\section{Welche Aussage zur Tuberkulose-Prävalenz unter}

\section{Flüchtlingen in Deutschland stimmt?}

A Flüchtlinge, die nach Deutschland kommen, haben keine Tuberkulose.

B Alle Flüchtlinge, die zu uns kommen, haben Tuberkulose.

C Die in einer hessischen Studie ermittelte Tuberkulose-Prävalenz unter den Flüchtlingen liegt niedriger, als es die WHO-Daten aus den Heimatländern erwarten lassen.

D Die Tuberkulose-Aufnahmeprävalenz unter den Flüchtlingen entspricht der Prävalenz in Deutschland.

E Bisherige Daten weisen auf eine Aufnahmeprävalenz von 305/100 000 Flüchtlingen hin.

3

Welche Aussage zur Tuberkulose bei Kindern ist richtig?

Erkrankte Kinder leiden v. a. unter Appetitlosigkeit und Gewichtsverlust. Nur wenige erkrankte Kinder sind symptomfrei.

C Eine hämatogene Aussaat der Tuberkulose ist v. a. für Säuglinge und Kleinkinder gefährlich.

D Kinder unter 10 Jahren sind besonders infektiös.

E Kinder können sich nicht mit Tuberkulose anstecken.

4 In welchem Fall ist - ohne Schutzmaßnahmen - eine Ansteckung mit Tbc am wahrscheinlichsten?

A nach Kontakt mit einem Patienten mit geschlossener Tuberkulose

B nach 10-minütigem Kontakt zu einem Patienten mit offener Lungen-Tbc ohne Husten

C nach mehrstündigem Besuch eines Kollegen, der am Tag zuvor Kontakt zu einem Tbc-Patienten hatte

D nach 30-minütiger Versorgung eines hustenden Erwachsenen mit offener Lungentuberkulose

E nach einem kurzen Gespräch mit einem hustenden 5-jährigen Mädchen, dessen Eltern an offener Lungentuberkulose leiden

5 Welche Tätigkeit ist nicht mit einem erhöhten Ansteckungsrisiko für Tuberkulose verbunden? Intubation

Absaugen

Reanimation

Hustenprovokation

Entsorgung eingetrockneten Sputums
Welche Reaktion nach einer möglichen Infektion mit Tbc im Dienst ist übertrieben?

Vorgesetzten informieren

betroffenen Mitarbeiter sofort aus dem Dienst nehmen

Ärztlichen Leiter Rettungsdienst konsultieren

Gedächtnisprotokoll erstellen

E vom Gesundheitsamt zum weiteren Vorgehen beraten lassen

Wann muss der Rettungsdienst eine Tuberkuloseinfektion behördlich melden?

nie

immer

nur, wenn akute Infektionsgefahr besteht

wenn der Patient nicht an eine ärztliche Einrichtung übergeben wird

Das ist in jedem Bundesland anders geregelt.

Wie viele Patienten erkranken nach einer Infektion mit Tuberkulose tatsächlich an der Krankheit?

$5-10 \%$

$25 \%$

$50 \%$

$85 \%$

$100 \%$

Wie wird Tuberkulose am häufigsten übertragen?

über Aerosole

fäkal-oral

durch Tierbisse

durch Bluttransfusionen

über Wundesekrete

Welcher Aspekt steht beim Transport eines TuberkulosePatienten im Vordergrund?

regelmäßige Desinfektion während der Fahrt

der Atemschutz

schnelles Fahren

die Lufttemperatur im Rettungswagen

E Patientenbereich im Wagen soweit wie möglich ausräumen

\section{cee.thieme.de}

- Sammeln Sie CEE-Punkte unter cee.thieme.de für Ihre Rettungsdienstfortbildung. 1 CEE-Punkt entspricht einer Fortbildungsstunde von 60 Minuten. 